Davide La Torre, University of Milan, Faculty of Political Sciences, via Conservatorio,7, 20122 Milano, Italy. e-mail: davide.latorre@unimi.it

Matteo Rocca, University of Insubria, Faculty of Economics, via Ravasi, 2, 21100 Varese, Italy. e-mail: matteo.rocca@uni-bocconi.it

\title{
HIGHER ORDER UNIFORM SMOOTHNESS AND DIFFERENTIABILITY OF REAL FUNCTIONS
}

\begin{abstract}
It is known that smoothness-type conditions have several implications on continuity and differentiability properties of real functions. When these conditions hold uniformly on an interval the implications become even stronger. The aim of this paper is to extend to higher orders the relations between uniform smoothness-type conditions and differentiability, taking into account higher order divided differences.
\end{abstract}

\section{Introduction}

A function $f:(a, b) \rightarrow \mathbb{R}$ is said to satisfy a smoothness-type condition at the point $x \in(a, b)$ when there exists a function $\phi$ converging to 0 as $h \rightarrow 0$, such that

$$
\frac{f(x+h)-2 f(x)+f(x-h)}{\phi(h)}=O(1)
$$

as $h \rightarrow 0$ (see for instance [17]). More specifically if

$$
\frac{f(x+h)-2 f(x)+f(x-h)}{h}=o(1)
$$

as $h \rightarrow 0$, then $f$ is said to be "smooth" at the point $x$, while if

$$
\frac{f(x+h)-2 f(x)+f(x-h)}{h}=O(1)
$$

as $h \rightarrow 0$, then $f$ is said to be "quasi-smooth" at $x$. If condition (1) (resp. (2)) holds at every point $x$ of an interval $(a, b)$, then $f$ is said to be smooth

Key Words: Hölder functions, divided differences

Mathematical Reviews subject classification: 26A24, 26A16

Received by the editors July 21, 2000 
(resp. quasi-smooth) on $(a, b)$. It is known that smoothness-type conditions have strong implications on the differentiability properties of a function. For a review of classical results one can see [17]. In particular we recall the following theorems.

Theorem 1.1. [17] If $f$ is a continuous smooth function on an interval $(a, b)$, then $f$ is differentiable on a set which is of the power of the continuum in any subinterval of $(a, b)$.

The previous result cannot be extended to quasi-smooth functions [17]. Anyway, we have the following:

Theorem 1.2. [17] Let $f$ be a measurable function and suppose that for some $\alpha \in(0,1]$ one has $\frac{f(x+h)-2 f(x)+f(x-h)}{h^{1+\alpha}}=O(1)$ as $h \rightarrow 0^{+}$, at every point in a measurable set $E$. Then $f$ is differentiable at almost every point of E.

When smoothness type conditions hold uniformly with respect to $x \in(a, b)$, they have stronger implications for what concerns continuity and differentiability of a function as we will see with Theorems 1.3 and 1.4 below.

Definition 1.1. A function $f:(a, b) \rightarrow \mathbb{R}$ is locally Hölder of degree $\alpha \in(0,1]$ at $x_{0} \in(a, b)$ when there exist a constant $K$ and a neighborhood $U$ of $x_{0}$ such that:

$$
|f(x)-f(y)| \leq K|x-y|^{\alpha} \text {, whenever } x, y \in U \text {. }
$$

Definition 1.2. A function $f:(a, b) \rightarrow \mathbb{R}$ is of class $C^{k, \alpha}$ at $x_{0} \in(a, b)$ when $f^{(k)}$ exists in a neighborhood of $x_{0}$ and $f^{(k)}$ is locally Hölder of degree $\alpha$ at $x_{0}\left(\right.$ we set $\left.f^{(0)}=f\right)$.

Theorem 1.3. [17] Assume that the function $f:(a, b) \rightarrow \mathbb{R}$ is bounded on a neighborhood of the point $x_{0} \in(a, b)$ and let $\alpha \in(0,1]$. If there exists a neighborhood $U$ of $x_{0} \in(a, b)$ such that $\frac{f(x+h)-2 f(x)+f(x-h)}{h^{1+\alpha}}=O(1)$ as $h \rightarrow 0^{+}$, uniformly with respect to $x \in U$, then $f$ is of class $C^{1, \alpha}$ at $x_{0}$.

It is known [17] that the previous theorem does not hold when $\alpha=0$. In this case we have the following.

Theorem 1.4. [17] Assume that the function $f:(a, b) \rightarrow \mathbb{R}$ is bounded on a neighborhood of the point $x_{0} \in(a, b)$. If on a neighborhood $U$ of the point $x_{0} \in(a, b) f$ satisfies the condition $\frac{f(x+h)-2 f(x)+f(x-h)}{h}=O(1)$ as $h \rightarrow 0^{+}$, uniformly with respect to $x \in U$, then $f$ is of class $C^{0, \alpha}$ at $x_{0}$ for every $\alpha \in(0,1)$. 
The aim of this paper is to extend to higher orders Theorems 1.3 and 1.4 (in the case $\alpha=1$, an extension to higher orders of Theorem 1.3 has been obtained by the authors in [13]). Section 2 is devoted to preliminary concepts and results, while in section 3 we give the main results.

\section{Preliminary Tools}

\subsection{Divided Differences, Peano Derivatives and $k$-Convex Func- tions}

In the following we will consider a function $f:(a, b) \rightarrow \mathbb{R}$. For such a function we put

$$
\Delta_{k} f(x ; h)=\sum_{i=0}^{k}(-1)^{k-i}\left(\begin{array}{l}
k \\
i
\end{array}\right) f\left(x+i h-\frac{1}{2} k h\right) .
$$

Besides this expression, in the proof of Theorem 3.1, we will consider differences $\tilde{\Delta}_{k} f(x ; h)$ defined recursively as follows:

$$
\tilde{\Delta}_{1} f(x ; h)=f(x+h)-f(x), \tilde{\Delta}_{k} f(x ; h)=\tilde{\Delta}_{k-1} f(x ; 2 h)-2^{k-1} \tilde{\Delta}_{k-1} f(x ; h) .
$$

As observed in [14], we have

$\tilde{\Delta}_{k} f(x ; h)=a_{k} f\left(x+2^{k-1} h\right)+a_{k-1} f\left(x+2^{k-2} h\right)+\cdots+a_{1} f(x+h)+a_{0} f(x)$,

where, for any fixed $k, a_{j}$ depends only on $j(j=0,1, \ldots, k-1)$ and $a_{k}=1$.

Lemma 2.1. [14] There are constants $C_{0}, C_{1}, \ldots, C_{2^{k-1}-k}$ such that:

$$
\tilde{\Delta}_{k} f(x ; h)=\sum_{i=0}^{2^{k-1}-k} C_{i} \Delta_{k} f\left(x+\frac{1}{2} k h+i h ; h\right) .
$$

The proof of the following lemma is straightforward from the previous result.

Lemma 2.2. If there exist a neighborhood $U$ of the point $x_{0}$ and a number $\alpha \in[0,1]$ such that $\frac{\Delta_{k} f(x ; h)}{h^{k-1+\alpha}}=O(1)$ as $h \rightarrow 0^{+}$, uniformly with respect to $x \in U$, then there exists a neighborhood $U^{\prime}$ of $x_{0}$ such that $\frac{\tilde{\Delta}_{k} f(x ; h)}{h^{k-1+\alpha}}=O(1)$ as $h \rightarrow 0^{+}$, uniformly with respect to $x \in U^{\prime}$.

Lemma 2.3. Assume that $f$ is bounded in a neighborhood of the point $x_{0}$. 
(i) If there exist a neighborhood $U$ of the point $x_{0}$ and a number $\alpha \in(0,1]$ such that $\frac{\tilde{\Delta}_{k} f(x ; h)}{h^{k-1+\alpha}}=O(1)$ as $h \rightarrow 0^{+}$, uniformly with respect to $x \in U$, then we also have $\frac{\tilde{\Delta}_{k-1} f(x ; h)}{h^{k-1}}=O(1)$ as $h \rightarrow 0^{+}$, uniformly with respect to $x \in U$.

(ii) If there exists a neighborhood $U$ of the point $x_{0}$ such that $\frac{\tilde{\Delta}_{k} f(x ; h)}{h^{k-1}}=O(1)$ as $h \rightarrow 0^{+}$, uniformly with respect to $x \in U$, then, for every $\alpha \in[0,1)$ we also have $\frac{\tilde{\Delta}_{k-1} f(x ; h)}{h^{k-2+\alpha}}=O(1)$ as $h \rightarrow 0^{+}$, uniformly with respect to $x \in U$.

Proof. (i) The proof is analogous to that of Lemma 2.3 in [13] and we omit it.

(ii) From the hypotheses we obtain the existence of numbers $\delta>0$ and $M \geq 0$ such that $\forall x \in U$ and $\forall h \in(0, \delta)$

$$
\begin{aligned}
\left|\tilde{\Delta}_{k-1} f(x ; h)-2^{k-1} \tilde{\Delta}_{k-1} f(x ; h / 2)\right| & \leq M(h / 2)^{k-1}, \\
\left|\tilde{\Delta}_{k-1} f(x ; h / 2)-2^{k-1} \tilde{\Delta}_{k-1} f(x ; h / 4)\right| & \leq M(h / 4)^{k-1}, \ldots \\
\left|\tilde{\Delta}_{k-1} f\left(x ; h / 2^{n-1}\right)-2^{k-1} \tilde{\Delta}_{k-1} f\left(x ; h / 2^{n}\right)\right| & \leq M\left(h / 2^{n}\right)^{k-1} .
\end{aligned}
$$

Multiplying these inequalities by $1,2^{k-1}, 2^{2(k-1)}, \ldots, 2^{(n-1)(k-1)}$ respectively, by addition we obtain

$$
\left|\tilde{\Delta}_{k-1} f(x ; h)-2^{n(k-1)} \tilde{\Delta}_{k-1} f\left(x ; h / 2^{n}\right)\right| \leq M n(h / 2)^{k-1},
$$

and hence $\left|2^{n(k-1)} \tilde{\Delta}_{k-1} f\left(x ; h / 2^{n}\right)\right| \leq M n(h / 2)^{k-1}+M^{\prime}$, for $\frac{1}{2} \delta \leq h \leq \delta$, by using the boundedness of $f$. Hence, writing $\xi=h / 2^{n}$, for every $x \in U$ we have $\left|\tilde{\Delta}_{k-1} f(x ; \xi)\right| \leq \frac{M n \xi^{k-1}}{2^{k-1}}+\frac{M^{\prime}}{2^{n(k-1)}}$ for $\xi \in\left(\delta / 2^{n+1}, \delta / 2^{n}\right), \quad n=$ $0,1, \ldots$ Since $\delta / 2^{n+1} \leq \xi \leq \delta / 2^{n}$ we obtain that $n=O(\log \xi)$ and hence for $x \in U$ we get $\left|\tilde{\Delta}_{k-1} f(x ; \xi)\right| \leq O\left(\xi^{k-1} \log \xi\right)+\frac{M^{\prime} \xi^{k-1}}{(\delta / 2)^{k-1}}$. Hence we obtain that $\frac{\tilde{\Delta}_{k-1} f(x ; \xi)}{\xi^{k-1} \log \xi}=O(1)$, as $\xi \rightarrow 0^{+}$, uniformly with respect to $x \in U$. The assertion now follows observing that for every $\alpha \in[0,1)$ we have $|\log \xi| \leq$ $|\xi|^{\alpha-1}$, for $\xi$ "small enough". 
Definition 2.1. If there exist numbers $f_{1}(x), \ldots, f_{k}(x)$ such that

$$
f(x+h)=f(x)+f_{1}(x) h+\frac{1}{2} f_{2}(x) h^{2}+\cdots+\frac{1}{k !} f_{k}(x) h^{k}+o\left(h^{k}\right),
$$

where $o\left(h^{k}\right) / h^{k} \rightarrow 0$ as $h \rightarrow 0$, then $f$ is said to admit a $k$-th Peano derivative at $x$. The number $f_{k}(x)$ is said the $k$-th Peano derivative of $f$ at $x$.

We say that $f$ admits $k$-th Peano derivative on an interval when it admits $k$-th Peano derivative at any point of this interval. It is well known that the existence of the ordinary $k$-th derivative of $f$ at $x, f^{(k)}(x)$, implies the existence of $f_{k}(x)$.

Lemma 2.4. [14] If $f_{k}(x)$ exists, then so does $\lim _{h \rightarrow 0} \frac{\tilde{\Delta}_{k} f(x ; h)}{h^{k}}$ and there exists a number $\lambda_{k}$, depending only on $k$, such that $\lambda_{k} \lim _{h \rightarrow 0} \frac{\tilde{\Delta}_{k} f(x ; h)}{h^{k}}=$ $f_{k}(x)$.

For a survey on Peano derivatives one can see for instance [6], [11], [15] and [18]. Further properties of Peano derivatives are given in [8], [9] and [10]. In particular we recall the following result.

Theorem 2.1. [15] If $f_{k}$ exists and is bounded (above or below) on an interval, then $f_{k}$ coincides with the ordinary derivative $f^{(k)}$ on this interval.

Definition 2.2. A continuous function $f:(a, b) \rightarrow \mathbb{R}$ is said to be $k$-convex when $\frac{\Delta_{k+1} f(x ; h)}{h^{k+1}} \geq 0$, for every $x \in(a, b)$ and for every $h$ such that $x \pm$ $\frac{k+1}{2} h \in(a, b)$.

Remark 2.1. If $f$ is not continuous the definition of $k$-convexity must be given considering divided differences at arbitrary (not equally spaced) points (see [2] for details).

When $k=1$ the previous definition reduces to that of convex function.

Theorem 2.2. [2] Let $f:(a, b) \rightarrow \mathbb{R}$ be a $k$-convex function.

(i) For $1 \leq r \leq k-1$ the derivative $f^{(r)}$ exists and is continuous in $(a, b)$ and furthermore $f_{+}^{(k)}$ exists on $(a, b)$ and is increasing (we denote by $f_{+}^{(k)}$ the $k$-th right derivative). 
(ii) If $h>0 ; x, t, y \in(a, b)$ and $y+\frac{k}{2} h \leq t \leq x$, then

$$
\frac{\Delta_{k} f(y ; h)}{h^{k}} \leq f_{+}^{(k)}(t) \leq \frac{\Delta_{k} f(x ; h)}{h^{k}} .
$$

(iii) The function $\frac{\Delta_{k} f(x, h)}{h^{k}}$ is increasing in $h$.

Theorem 2.3. [2] Let $f:(a, b) \rightarrow \mathbb{R}$ be a function that admits $f_{+}^{(k)}$ on $(a, b)$. Then $f$ is $k$-convex if and only if $f_{+}^{(k)}$ is increasing on $(a, b)$.

Lemma 2.5. Assume that the function $f:(a, b) \rightarrow \mathbb{R}$ is continuous and $k$-convex on a neighborhood $\left(x_{0}-\varepsilon, x_{0}+\varepsilon\right)$ of the point $x_{0} \in(a, b)$ and let $\varepsilon^{\prime} \in(0, \varepsilon)$. For every $x \in\left(x_{0}-\varepsilon^{\prime}, x_{0}+\varepsilon^{\prime}\right)$ and $h \in\left(0,\left(\varepsilon-\varepsilon^{\prime}\right) / k\right)$, there exists a point $\xi \in\left(x_{0}-\varepsilon, x_{0}+\varepsilon\right)$ such that $\frac{\Delta_{k} f(\xi ; h)}{h^{k}}=f_{+}^{(k)}(x)$.

Proof. It is always possible to find $y \in\left(x_{0}-\varepsilon, x_{0}+\varepsilon\right)$ such that $y+\frac{k}{2} h<x$. From Theorem 2.2 it follows that $\frac{\Delta_{k} f(y ; h)}{h^{k}} \leq f_{+}^{(k)}(x) \leq \frac{\Delta_{k} f(x ; h)}{h^{k}}$. Since $\frac{\Delta_{k} f(x ; h)}{h^{k}}$ is continuous as a function of $x$, the assertion follows from the Darboux Theorem.

\subsection{Standard Mollifiers}

The function $\phi$, defined by

$$
\phi(x)= \begin{cases}C \exp \left(\frac{1}{x^{2}-1}\right) & \text { if }|x|<1 \\ 0 & \text { if }|x| \geq 1\end{cases}
$$

is $C^{\infty}(\mathbb{R})$ and we can choose the constant $C \in \mathbb{R}$ such that $\int_{\mathbb{R}} \phi(x) d x=1$.

Definition 2.3. Let $\varepsilon>0$. The functions $\phi_{\varepsilon}(x)=\frac{\phi\left(\frac{x}{\varepsilon}\right)}{\varepsilon}$ are called standard mollifiers.

Definition 2.4. Let $f:(a, b) \rightarrow \mathbb{R}$. We say that $f \in C_{0}^{k}((a, b))$ if $f \in$ $C^{k}((a, b))$ and

$$
\operatorname{spt}_{f}=\overline{\{x \in(a, b): f(x) \neq 0\}} \subset(a, b) .
$$

Theorem 2.4. [1] The functions $\phi_{\varepsilon}$ are $C^{\infty}(\mathbb{R})$ and satisfy 
(i) $\int_{\mathbb{R}} \phi_{\varepsilon}(x) d x=1$

(ii) $\operatorname{spt}_{\phi_{\varepsilon}} \subset B(0, \varepsilon)$.

For $f \in L_{\text {loc }}^{1}(a, b)$, and $\varepsilon>0$ we define functions $f_{\varepsilon}$ by the convolution $f_{\varepsilon}(x)=\int_{a}^{b} \phi_{\varepsilon}(x-y) f(y) d y$. Since $\phi_{\varepsilon}(x)$ is an even function, we can also write $f_{\varepsilon}(x)=\int_{a}^{b} \phi_{\varepsilon}(y-x) f(y) d y$. Observe that $f_{\varepsilon}(x)=0$ if $x \in \mathbb{R} \backslash[a-\varepsilon, b+\varepsilon]$ and that $f_{\varepsilon} \in C^{\infty}(\mathbb{R})$.

Theorem 2.5. [1] Suppose that $f \in L_{l o c}^{1}(a, b)$. Then for a.e. $x \in(a, b)$ we have $f_{\varepsilon}(x) \rightarrow f(x)$, as $\varepsilon \rightarrow 0^{+}$. If $f \in C((a, b))$, then the convergence is uniform on compact subsets of $(a, b)$.

Theorem 2.6. [1] Let $[c, d] \subset(a, b)$. Then $\exists \varepsilon_{0}>0$ such that $\forall \varepsilon \in\left(0, \varepsilon_{0}\right)$ and $\forall x \in[c, d]$ the function $y \rightarrow \phi_{\varepsilon}(x-y)$ is $C_{0}^{\infty}((a, b))$.

\section{Higher Order Uniform Smoothness and Differentiabil- ity}

In this section we extend Theorems 1.3 and 1.4 to higher orders.

Theorem 3.1. Assume that the function $f:(a, b) \rightarrow \mathbb{R}$ is bounded on a neighborhood of the point $x_{0} \in(a, b)$, let $\alpha \in(0,1]$ and $k \geq 2$. If there exists a neighborhood $U$ of $x_{0}$ such that $\frac{\Delta_{k+1} f(x ; h)}{h^{k+\alpha}}=O(1)$ as $h \rightarrow 0^{+}$, uniformly with respect to $x \in U$, then $f$ is of class $C^{k, \alpha}$ at $x_{0}$.

Proof. From the hypotheses we deduce the existence of a right neighborhood $V_{+}$of the origin such that $\frac{\Delta_{k+1} f(x ; h)}{h^{k+\alpha}}$ is bounded on $U \times V_{+} \backslash\{0\}$. Hence from Lemmas 2.2 and 2.3 (i) we obtain the existence of a neighborhood $U^{\prime}$ of $x_{0}$ and a right neighborhood $V_{+}^{\prime}$ of 0 such that $\frac{\tilde{\Delta}_{j} f(x ; h)}{h^{j}}$ are bounded on $U^{\prime} \times$ $V_{+}^{\prime} \backslash\{0\}, \forall j=1, \ldots, k$. Observe that the boundedness of $\frac{\tilde{\Delta}_{1} f(x ; h)}{h}$ means that $f$ is locally Lipschitz at the point $x_{0}$ and hence continuous in a neighborhood of $x_{0}$. For every $x$ in a neighborhood of $x_{0}$ and for $\varepsilon$ "sufficiently small", recalling Lemma 2.4 and Theorem 2.6, and using the Lebesgue convergence 
Theorem, for $1 \leq j \leq k$ we have

$$
\begin{aligned}
f_{\varepsilon}^{(j)}(x) & =(-1)^{j} \int_{a}^{b} \phi_{\varepsilon}^{(j)}(y-x) f(y) d y \\
& =(-1)^{j} \lambda_{j} \int_{a}^{b} \lim _{h \rightarrow 0^{+}} \frac{\tilde{\Delta}_{j} \phi_{\varepsilon}(y-x ; h)}{h^{j}} f(y) d y \\
& =(-1)^{j} \lambda_{j} \int_{a}^{b} \lim _{h \rightarrow 0^{+}} \frac{\sum_{i=1}^{j} a_{i} \phi_{\varepsilon}\left(y-x+2^{i-1} h\right)+a_{0} \phi_{\varepsilon}(y-x)}{h^{j}} f(y) d y \\
& =(-1)^{j} \lambda_{j} \lim _{h \rightarrow 0^{+}} \int_{a}^{b} \frac{\sum_{i=1}^{j} a_{i} \phi_{\varepsilon}\left(y-x+2^{i-1} h\right)+a_{0} \phi_{\varepsilon}(y-x)}{h^{j}} f(y) d y
\end{aligned}
$$

Now, putting $z=y+2^{i-1} h$, we obtain

$$
\int_{a}^{b} \frac{a_{i} \phi_{\varepsilon}\left(y-x+2^{i-1} h\right)}{h^{j}} f(y) d y=\int_{a+2^{i-1} h}^{b+2^{i-1} h} \frac{a_{i} f\left(z-2^{i-1} h\right) \phi_{\varepsilon}(z-x)}{h^{j}} d z .
$$

Thus

$$
\begin{aligned}
& (-1)^{j} \lambda_{j} \int_{a}^{b} \frac{\sum_{i=1}^{j} a_{i} \phi_{\varepsilon}\left(y-x+2^{i-1} h\right)+a_{0} \phi_{\varepsilon}(y-x)}{h^{j}} f(y) d y \\
= & (-1)^{j} \lambda_{j} \sum_{i=1}^{j} \int_{a+2^{i-1} h}^{b+2^{i-1} h} \frac{a_{i} f\left(z-2^{i-1} h\right) \phi_{\varepsilon}(z-x)}{h^{j}} d z \\
& +(-1)^{j} \lambda_{j} \int_{a}^{b} \frac{a_{0} f(z) \phi_{\varepsilon}(z-x)}{h^{j}} d z .
\end{aligned}
$$

For $h<\frac{b-a}{2^{k-1}}$, from Theorem 2.6 for all "sufficiently small" $\varepsilon$, the previous equation is equal to

$$
\begin{aligned}
& (-1)^{j} \lambda_{j} \sum_{i=1}^{j} \int_{a}^{b} \frac{a_{i} f\left(z-2^{i-1} h\right) \phi_{\varepsilon}(z-x)}{h^{j}} d z+(-1)^{j} \lambda_{j} \int_{a}^{b} \frac{a_{0} f(z) \phi_{\varepsilon}(z-x)}{h^{j}} d z \\
& =(-1)^{j} \lambda_{j} \int_{a}^{b} \frac{\tilde{\Delta}_{j} f(z,-h)}{h^{j}} \phi_{\varepsilon}(z-x) d z=\lambda_{j} \int_{a}^{b} \frac{\tilde{\Delta}_{j} f(z,-h)}{(-h)^{j}} \phi_{\varepsilon}(z-x) d z .
\end{aligned}
$$

Hence we get $f_{\varepsilon}^{(j)}(x)=\lambda_{j} \lim _{h \rightarrow 0^{+}} \int_{a}^{b} \frac{\tilde{\Delta}_{j} f(z, h)}{h^{j}} \phi_{\varepsilon}(z-x) d z$.

From the boundedness of $\frac{\tilde{\Delta}_{j} f(x, h)}{h^{j}}$ we get the existence of a constant $M$ such that $\left|f_{\varepsilon}^{(j)}(x)\right| \leq M$, for every $\varepsilon$ "sufficiently small" and for every $x$ 
in a neighborhood of $x_{0}$. In this way we established that $f_{\varepsilon}^{(j)}(x)$ is bounded (uniformly with respect to $\varepsilon$ ) on a neighborhood of $x_{0}, \forall j=1, \ldots, k$. Hence, there exists a neighborhood $\tilde{U}$ of $x_{0}$ such that for $x \in \tilde{U}$ there is a sequence $\varepsilon_{n}$ converging to 0 such that for all $j=1, \ldots, k-1$, the sequence $f_{\varepsilon_{n}}^{(j)}(x)$ converges to a limit which we denote by $\alpha_{j}(x)$. Notice that the functions $\alpha_{j}(x), j=1, \ldots, k-1$, are bounded on $\tilde{U}$. The functions $f_{\varepsilon_{n}}(x)$ are of class $C^{\infty}$ and hence $\forall x, y \in \tilde{U}$

$f_{\varepsilon_{n}}(y)=f_{\varepsilon_{n}}(x)+f_{\varepsilon_{n}}^{\prime}(x)(y-x)+\cdots+\frac{f_{\varepsilon_{n}}^{(k-1)}(x)}{(k-1) !}(y-x)^{k-1}+\frac{f_{\varepsilon_{n}}^{(k)}\left(\xi_{n}\right)}{k !}(y-x)^{k}$,

where $\xi_{n} \in(x, y)$. Recalling Theorem 2.5, taking the limit for $n \rightarrow+\infty$ it follows that $f_{\varepsilon_{n}}^{(k)}\left(\xi_{n}\right)$ converges to a limit which we denote by $\beta(x, y)$. Moreover $f(y)=f(x)+\alpha_{1}(x)(y-x)+\cdots+\frac{1}{(k-1) !} \alpha_{k-1}(x)(y-x)^{k-1}+\frac{1}{k !} \beta(x, y)(y-x)^{k}$.

Observing that $\beta(x, y)$ is bounded for $x, y \in \tilde{U}$, we have that $\forall x \in \tilde{U}, \alpha_{k-1}(x)$ is the $(k-1)$-th Peano derivative of $f$ at $x$. From Theorem 2.1 it follows that $\alpha_{k-1}(x)=f^{(k-1)}(x), \forall x \in \tilde{U}$. Furthermore the functions $f_{\varepsilon}^{(k)}$ are bounded uniformly with respect to $\varepsilon$, for $\varepsilon$ "sufficiently small" and thus the functions $f_{\varepsilon_{n}}^{(k-1)}$ satisfy the uniform Lipschitz condition

$$
\left|f_{\varepsilon_{n}}^{(k-1)}(y)-f_{\varepsilon_{n}}^{(k-1)}(x)\right| \leq B|y-x|, \forall x, y \in \tilde{U} .
$$

Since $f_{\varepsilon_{n}}^{(k-1)}(x)$ and $f_{\varepsilon_{n}}^{(k-1)}(y)$ converge to $f^{(k-1)}(x)$ and $f^{(k-1)}(y)$ respectively, we see that $f^{(k-1)}$ is Lipschitzian on $\tilde{U}$.

For $\varepsilon$ "small enough", let $M$ be an upper bound for $f_{\varepsilon}^{(k)}$ on $\tilde{U}$ and consider the function $\tilde{f}(x)=f(x)+p(x)$, where $p(x)=c_{0}+c_{1} x+\cdots+c_{k} x^{k}$ is any polynomial of degree $k$, with $c_{k} k ! \geq-M$. With easy calculations we get $\tilde{f}_{\varepsilon}^{(k)}(x)=f_{\varepsilon}^{(k)}(x)+k ! c_{k} \geq 0$ for $x \in \tilde{U}$ and hence $\tilde{f}_{\varepsilon}^{(k-2)}(x)$ is convex on $\tilde{U}$ (we let $f^{(0)}=f$ ). For $x, y \in \tilde{U}$ and $t \in[0,1]$,

$$
\tilde{f}_{\varepsilon}^{(k-2)}(t x+(1-t) y) \leq t \tilde{f}_{\varepsilon}^{(k-2)}(x)+(1-t) \tilde{f}_{\varepsilon}^{(k-2)}(y)
$$

Sending $\varepsilon$ to 0 and recalling Theorem 2.5, we obtain that $\tilde{f}^{(k-2)}(x)$ is convex on $\tilde{U}$. It follows from Theorem 2.3 that $\tilde{f}$ is $(k-1)$-convex on $\tilde{U}$ and hence, by Lemma 2.5 , for $x$ in a suitable neighborhood of $x_{0}$ and $h$ "small enough" $(h \neq 0), \frac{\Delta_{k-1} \tilde{f}(\xi ; h)}{h^{k-1}}=\tilde{f}^{(k-1)}(x)$ for some $\xi \in \tilde{U}$. Now, using classical 
properties of differences $\Delta_{k}$, we have

$$
\frac{\Delta_{2} \tilde{f}^{(k-1)}(x ; h)}{h^{1+\alpha}}=\frac{\Delta_{2} \Delta_{k-1} \tilde{f}(\xi ; h)}{h^{k+\alpha}}=\frac{\Delta_{k+1} \tilde{f}(\xi ; h)}{h^{k+\alpha}}=\frac{\Delta_{k+1} f(\xi ; h)}{h^{k+\alpha}}
$$

and hence we get that $\frac{\Delta_{2} \tilde{f}^{(k-1)}(x ; h)}{h^{1+\alpha}}$ is bounded for $x$ in a neighborhood of $x_{0}$ and $h$ "sufficiently small". From $\tilde{f}^{(k-1)}(x)=f^{(k-1)}(x)+a_{k-1}(k-1) !+a_{k} k ! x$ we easily obtain $\frac{\Delta_{2} \tilde{f}^{(k-1)}(x ; h)}{h^{1+\alpha}}=\frac{\Delta_{2} f^{(k-1)}(x ; h)}{h^{1+\alpha}}$. To complete the proof it is enough to apply Theorem 1.3 to the function $f^{(k-1)}$.

Theorem 3.2. Assume that the function $f:(a, b) \rightarrow \mathbb{R}$ is bounded in $a$ neighborhood of the point $x_{0} \in(a, b)$. If there exists a neighborhood $U$ of $x_{0}$ such that that $\frac{\Delta_{k+1} f(x ; h)}{h^{k}}=O(1)$ as $h \rightarrow 0^{+}$, uniformly with respect to $x \in U$, hen $f$ is of class $C^{k-1, \alpha}$ at $x_{0}$, whenever $\alpha \in(0,1)$.

Proof. The proof is analogous to that of the previous theorem and we give only a sketch of it. From Lemma 2.2 , we get $\frac{\tilde{\Delta}_{k+1} f(x ; h)}{h^{k}}=O(1)$ as $h \rightarrow 0^{+}$, uniformly with respect to $x$ in a neighborhood $U^{\prime}$ of $x_{0}$. Using Lemma 2.3 (ii), we get $\frac{\tilde{\Delta}_{k} f(x ; h)}{h^{k-1+\alpha}}=O(1)$ as $h \rightarrow 0^{+}$, uniformly with respect to $x \in U^{\prime}$, whenever $\alpha \in(0,1)$. Now the proof follows in a fashion similar to that of Theorem 3.1, with the steps sketched below

(i) We prove that $f^{(k-2)}(x)$ exists and is bounded for $x$ in a neighborhood $\tilde{U}$ of $x_{0}$ and that $f_{\varepsilon}^{(k-1)}$ is bounded on $\tilde{U}$ (uniformly with respect to $\varepsilon$ ).

(ii) Let $M$ be an upper bound for the functions $f_{\varepsilon}^{(k-1)}$ on $\tilde{U}$. The function $\tilde{f}(x)=f(x)+p(x)$, where $p(x)=c_{0}+c_{1} x+\cdots+c_{k-1} x^{k-1}$ and $c_{k-1}(k-1) ! \geq$ $M$, is $(k-2)$-convex.

(iii) Recalling Lemma 2.5 we get that $\frac{\Delta_{2} f^{(k-2)}(x ; h)}{h^{1+\alpha}}=O(1)$ as $h \rightarrow 0^{+}$, uniformly with respect to $x$ in a neighborhood of $x_{0}$, whenever $\alpha \in(0,1)$.

(iv) Applying Theorem 1.3 to the function $f^{(k-2)}$ we get the assertion.

Remark 3.1. The requirement

$$
\frac{\Delta_{k+1} f(x ; h)}{h^{k}}=O(1), \text { as } h \rightarrow 0^{+}
$$


can be considered as an higher order quasi-smoothness condition for $f$ at $x$. Higher order smoothness and quasi-smoothness conditions of a different type have been investigated in [3], [4], [5] and [7], where the authors recall a definition introduced by Zygmund [19] (but the uniform case is not studied in these papers). Furthermore it can be easily seen that condition (3) is more general than the quasi-smoothness notion studied in the papers above.

\section{References}

[1] H. Brezis, Analyse fonctionelle- Theorie et applications. Masson Editeur, Paris, 1963.

[2] P. S. Bullen, A criterion for n-convexity. Pac. J. Math., 36 (1971), 81-98.

[3] Z. Buczolich, M. J. Evans, P. D. Humke, Approximate high order smoothness. Acta Math. Hung., 61 (1993), 369-388.

[4] T. K. Dutta, Generalized smooth functions. Acta Math. Acad. Sci. Hungar., 40 (1982), 29-37.

[5] T. K. Dutta, N. Mukhopadhyay, Generalized smooth functions II. Acta. Math. Hung., 55 (1990), 47-56.

[6] M. J. Evans, C. E. Weil, Peano derivatives: a survey. Real Analysis Exchange, 7 (1981-82), 5-23.

[7] M. J. Evans, High order smoothness. Acta Math. Hung., 50 (1987), 17-20.

[8] I. Ginchev, A. Guerraggio, M. Rocca, Equivalence of Peano and Riemann derivatives. Proceedings of the Workshop on Optimization and Generalized Convexity for economic applications, Verona, may 1998, (G. Giorgi and F.A. Rossi eds.)

[9] I. Ginchev, M. Rocca, On Peano and Riemann derivatives. Rendiconti del Circolo Matematico di Palermo, 49, (2000), 153-164.

[10] I. Ginchev, A. Guerraggio, M. Rocca, Equivalence of $(n+1)$-th order Peano and usual derivatives for n-convex functions. Real Analysis Exchange, 25 (2000), 513-520.

[11] A. Guerraggio, M. Rocca, Derivate dirette di Riemann e di Peano. Convessitá e Calcolo Parallelo (G. Giorgi and F.A. Rossi eds.), Verona, 1997.

[12] E. Isaacson, B. H. Keller, Analysis of numerical methods. Wiley, New York, 1966. 
[13] D. La Torre, M. Rocca, $C^{k, 1}$ functions and Riemann derivatives. Real Analysis Exchange, 25, (2000), 743-752.

[14] J. Marcinkiewicz, A. Zygmund, On the differentiability of functions and summability of trigonometrical series. Fund. Math., 26 (1936), 1-43.

[15] H. W. Oliver, The exact Peano derivative. Trans. Amer. Math.Soc., 76 (1954), 444-456.

[16] G. Peano, Sulla formula di taylor. Atti Accad. Sci. Torino, 27 (1981-92), 40-46.

[17] B. S. Thomson, Symmetric properties of real functions. Marcel Dekker, New York, 1994.

[18] C. E. Weil, The Peano notion of higher order differentiation. Math. Japon., 42 (1995), 587-600.

[19] A. Zygmund, Trigonometric Series. Cambridge University Press, Cambridge, 1959. 